\title{
INDICADORES BIOLÓGICOS DE QUALIDADE DO SOLO EM DIFERENTES SISTEMAS DE USO NO BREJO PARAIBANO ${ }^{1}$
}

\author{
Biological indicators of soil quality in different land use systems in paraiban swamp region, Brazil
}

\author{
Mônica Lima Pôrto ${ }^{2}$, Jailson do Carmo Alves ${ }^{3}$, Adriana Araújo Diniz ${ }^{4}$,Adailson Pereira de Souza ${ }^{5}$, Djail Santos ${ }^{5}$
}

\begin{abstract}
RESUMO
A avaliação da qualidade do solo é uma ferramenta importante para monitorar a sua degradação, bem como planejar a implantação de práticas sustentáveis de manejo. Neste trabalho, objetivou-se avaliar indicadores biológicos de qualidade do solo em um Latossolo Amarelo distrófico, submetido a diferentes sistemas de uso em Areia-PB. Foram utilizadas amostras de solo coletadas na camada arável $(0-20 \mathrm{~cm})$ em áreas de mata nativa, fruticultura, cana-de-açúcar, sucessão de cultivos, pastagem e consórcio de culturas. Foram avaliados o carbono orgânico total (COT), carbono da biomassa microbiana (CBM), quociente microbiano ( $q$ Mic), respiração basal (RB) e quociente metabólico $\left(q \mathrm{CO}_{2}\right)$. Os resultados obtidos indicaram que as áreas sob gramíneas apresentaram uma tendência de manutenção do COT e menores reduções no CBM, entretanto, apresentaram elevados valores de $q \mathrm{CO}_{2}$, indicando a ocorrência de um processo degradativo. A área sob fruticultura apresentou leves reduções no COT e CBM e baixos valores de $q \mathrm{CO}_{2}$, indicando que esse sistema pode estar se ajustando a um novo estado de equilíbrio. As áreas sob sucessão de cultivos e consórcio de culturas apresentaram as maiores reduções no COT e CBM e elevados valores de $q \mathrm{CO}_{2}$, demonstrando um elevado estágio de degradação desses sistemas. Pode-se concluir que todos os sistemas agrícolas estudados promoveram perda de qualidade do solo, sendo esse fato mais pronunciado nas áreas sob manejo mais intensivo. $\mathrm{O} \mathrm{CBM}$ e $q \mathrm{CO}_{2}$ mostraram-se bastantes sensíveis às alterações decorrentes do uso agrícola do solo, apresentando grande potencial para estudos de sua qualidade.
\end{abstract}

Termos para indexação: Agroecossistema, biomassa microbiana, atividade microbiana, quociente microbiano, quociente metabólico.

\begin{abstract}
Soil quality evaluation is an important tool for the monitoring of soil degradation as well as for planning the adoption of sustainable agricultural management practices. The objective of this study was to evaluate some biological indicators of the soil quality of a dystrophic Yellow Latosol (Oxisol) under different land use systems in Areia, Paraíba, Brazil. Soil samples were taken form the plow layer $(0-20 \mathrm{~cm})$ at six sites: native vegetation (moist Atlantic forest), fruit culture (cashew crop), sugarcane, crop succession (sorghum/common bean/castor bean), pasture (Brachiaria decumbens), and a combined cropping system (castor bean + sorghum). Total organic carbon (TOC), microbial biomass carbon (MBC), microbial quotient ( $q$ Mic), basal respiration (BR) and metabolic quotient $\left(q \mathrm{CO}_{2}\right)$ were evaluated. The results indicated that treatments under grasses, such as sugarcane and pasture, showed a tendency to maintain the TOC contents with lower reductions in $\mathrm{MBC}$. However, higher $q \mathrm{CO}_{2}$ values were found under these treatments, which may indicate the occurrence of degradation processes. Slight reductions in TOC and MBC values and low $q \mathrm{CO}_{2}$ values were verified in the cashew crop, suggesting that the soil under this system could be adjusting to a new equilibrium state. Crop succession and combined cropping system treatments had the largest reductions in TOC and MBC with the highest $q \mathrm{CO}_{2}$ values, which suggests a stage of advanced degradation. It can be concluded that all the agroecosystems evaluated in this study resulted in loss of soil quality, which was pronounced in the most intensively managed treatments. The $\mathrm{MBC}$ and $q \mathrm{CO}_{2}$ were highly sensitive to land use changes and show great potential for soil quality evaluations.
\end{abstract}

Index terms: Agroecosystem, microbial biomass, microbial activity, microbial quotient, metabolic quotient.

(Recebido em 4 de outubro de 2007 e aprovado em 19 de setembro de 2008)

\section{INTRODUÇÃO}

Os atributos biológicos do solo podem ser considerados indicadores de alguns processos que ocorrem no solo em resposta às perturbações antropogênicas, podendo constituir-se importantes variáveis para predizer a qualidade dos ecossistemas agrícolas. Apesar disso, estudos de resposta microbiológica de sistemas edáficos a intervenções antrópicas são relativamente escassos para as condições do Nordeste brasileiro.

\footnotetext{
${ }^{1}$ Trabalho apresentado no XXXI CBCS em Gramado-RS no período de 05 a 11 de agosto de 2007.

${ }^{2}$ Eng. Agrônoma, Doutoranda do PPG Fitotecnia, Departamento de Fitotecnia, Universidade Federal de Viçosa, Avenida P.H. Rolfs, s/n, Viçosa, MG, CEP 36.570-000. Bolsista do CNPq. E-mail: monicalporto@yahoo.com.br.

${ }^{3}$ Eng. Agrônomo, Doutorando do PPG Solos e Nutrição de Plantas, Departamento de Solos, Universidade Federal de Viçosa, Avenida P.H. Rolfs, s/n, Viçosa, MG, CEP 36.570-000. Bolsista do CNPq. E-mail: jailson_agro@yahoo.com.br.

${ }^{4}$ Eng. Agrônoma, Doutoranda do PPG Agronomia, Centro de Ciências Agrárias (CCA), Universidade Federal da Paraíba (UFPB), Areia, PB, CEP 58.397-000. Bolsista da CAPES. E-mail: adrisolos@bol.com.br.

${ }^{5}$ Eng. Agrônomo, D. Sc., Prof. Adjunto do Departamento de Solos e Engenharia Rural, CCA/UFPB, Areia, PB, CEP 58.397-000 - adailson@cca.ufpb.br; santosdj@cca.ufpb.br
} 
Os microrganismos do solo apresentam papel fundamental na decomposição da matéria orgânica, formação e estabilização de agregados e ciclagem biogeoquímica de nutrientes no solo. A sua biomassa representa ainda uma expressiva parcela do carbono $(\mathrm{C})$, nitrogênio $(\mathrm{N})$ e fósforo (P) do solo (Nannipieri et al., 2003; Pokarzhevskii et al., 2003). Além disso, pesquisas recentes têm apontado que alguns indicadores relacionados com a comunidade microbiana do solo são bastante sensíveis às alterações provenientes das atividades agrícolas, fornecendo, dessa forma, subsídios importantes para o correto planejamento do uso da terra e manejo do solo (Johnson et al., 2003; Matsuoka et al., 2003; Sangha et al., 2005).

O tamanho, composição e atividade da microbiota do solo têm sido frequentemente utilizados em estudos de monitoramento das alterações ambientais decorrentes da exploração agrícola. No entanto, verifica-se que essas variáveis isoladamente não expressam adequadamente os processos biogeoquímicos que ocorrem nos ecossistemas, devendo ser combinados entre si, de forma a produzir relações (Anderson, 2003; Harris, 2003). Por exemplo, trabalhos têm demonstrado que os quocientes microbianos $(q \mathrm{Mic})$ e metabólicos $\left(q \mathrm{CO}_{2}\right)$ são sensíveis aos efeitos ambientais e antropogênicos sobre a comunidade microbiana do solo, podendo constituir-se indicadores das perturbações dos ecossistemas (Andréa et al., 2002; Harris, 2003).

O emprego de sistemas de cultivo e práticas de manejo do solo como o monocultivo, aração e gradagem, aplicações de agrotóxicos, adubos e corretivos, entre outras, podem desequilibrar a microbiota, reduzindo a diversidade dos microrganismos e a ciclagem de nutrientes, podendo resultar em degradação do solo e menor produtividade das culturas (Jordan et al., 2004; Díaz-Raviña et al., 2005). As diferentes culturas agrícolas, em razão de apresentarem características distintas (ciclo de vida, ambiente de rizosfera, produção e deposição de material vegetal sobre e dentro do solo, entre outras) e exigências específicas (preparo do solo, adubação, irrigação, podas, entre outras), promovem influências e alterações distintas sobre a biomassa microbiana do solo e sua atividade (Johnson et al., 2003; Matsuoka et al., 2003).

Neste trabalho, objetivou-se avaliar indicadores biológicos de qualidade de um Latossolo Amarelo distrófico submetido a diferentes sistemas de uso em Areia-PB.

\section{MATERIAL E MÉTODOS}

O trabalho foi conduzido no período de outubro a novembro de 2006, no Laboratório de Biotecnologia do Solo do Departamento de Solos e Engenharia Rural (DSER) do Centro de Ciências Agrárias (CCA) da Universidade Federal da Paraíba (UFPB) em Areia-PB, utilizando amostras de um Latossolo Amarelo distrófico submetido a diferentes sistemas de uso (Tabela 1). As amostras de solo foram coletadas em outubro de 2006, na Fazenda Experimental Chã de Jardim, pertencente ao CCA/ UFPB. A área experimental está localizada em Areia-PB (microrregião do Brejo Paraibano), nas coordenadas geográficas $6^{\circ} 58^{\prime} 12^{\prime}$ ' S e $35^{\circ} 42^{\prime} 15^{\prime}$ ' W. O clima local, de acordo com a classificação de Köppen, é do tipo As' (quente e úmido), com precipitação pluviométrica média anual em torno de $1500 \mathrm{~mm}$, temperatura média anual oscilando entre 22 a $26^{\circ} \mathrm{C}$ e umidade relativa do ar elevada (75 a $87 \%$ ) (Brasil, 1972).

O trabalho foi conduzido em um delineamento inteiramente casualizado, com seis tratamentos (sistemas de uso do solo) e cinco repetições. Para cada sistema de uso do solo, obteve-se uma amostra composta a partir da coleta de dez amostras simples na camada de 0-20 cm. As amostras foram homogeneizadas, secas ao ar, destorroadas e passadas em peneiras com malha de $2 \mathrm{~mm}$. Posteriormente, uma parte foi encaminhada para o Laboratório de Química e Fertilidade do Solo do DSER/CCA/UFPB para a sua caracterização química (Embrapa, 1997) (Tabela 2) e o restante para o Laboratório de Biotecnologia do Solo para as determinações de natureza biológica (carbono orgânico total, carbono da biomassa microbiana, quociente microbiano, respiração basal e quociente metabólico).

Tabela 1 - Histórico de utilização de um Latossolo Amarelo distrófico submetido a diferentes sistemas de uso em Areia-PB.

\begin{tabular}{lcl}
\hline \multicolumn{1}{c}{ Sistemas de Uso } & Símbolo & \multicolumn{1}{c}{ Histórico de Utilização da Área } \\
\hline Mata Nativa & MATA & Remanescente de Mata Atlântica \\
Fruticultura & FRUT & Cultivada com a cultura do caju desde 1995 \\
Cana-de-Açúcar & CANA & Introduzida em 1993 \\
Sucessão de Cultivos & SUCE & Sucessão sorgo/feijão/mamona \\
Pastagem & PAST & Cultivada com Brachiaria decumbens desde 2000 \\
Consórcio de Culturas & CONS & Consórcio mamona + sorgo desde 2003 \\
\hline
\end{tabular}


Tabela 2 - Caracterização química inicial de um Latossolo Amarelo distrófico submetido a diferentes sistemas de uso em Areia-PB.

\begin{tabular}{|c|c|c|c|c|c|c|c|c|}
\hline \multirow[b]{2}{*}{ Sistemas de Uso } & \multicolumn{8}{|c|}{ Características } \\
\hline & $\begin{array}{c}\mathrm{pH} \\
\left(\mathrm{H}_{2} \mathrm{O} 1: 2,5\right)\end{array}$ & $\mathrm{P}$ & $\mathrm{K}$ & $\mathrm{Ca}$ & $\mathrm{Mg}$ & $\mathrm{H}+\mathrm{Al}$ & SB & CTC \\
\hline & \multicolumn{4}{|c|}{$--\mathrm{mg} \mathrm{dm}^{-3}--$} & \multicolumn{4}{|c|}{ - $\mathrm{cmol}_{\mathrm{c}} \mathrm{dm}^{-3}$} \\
\hline MATA & 4,1 & 3,65 & 32,96 & 1,05 & 0,50 & 14,36 & 1,67 & 16,03 \\
\hline FRUT & 5,1 & 3,00 & 43,88 & 2,85 & 0,80 & 8,75 & 3,80 & 12,54 \\
\hline CANA & 5,4 & 3,65 & 89,12 & 3,15 & 1,70 & 6,44 & 5,12 & 11,55 \\
\hline SUCE & 5,1 & 1,70 & 18,92 & 2,45 & 0,50 & 7,10 & 3,07 & 10,16 \\
\hline PAST & 5,3 & 2,35 & 28,28 & 0,65 & 1,00 & 11,23 & 1,78 & 12,01 \\
\hline CONS & 5,9 & 1,70 & 18,92 & 2,85 & 2,60 & 7,53 & 5,54 & 13,05 \\
\hline
\end{tabular}

O carbono orgânico total (COT) do solo foi determinado por meio de oxidação da matéria orgânica com dicromato de potássio 0,2 M, em meio ácido (Embrapa, 1997).

Para a determinação do carbono da biomassa microbiana (CBM) do solo foi empregado o método de fumigação incubação, sendo adotados os procedimentos descritos por Jenkinson \& Powlson (1976), com modificações. A umidade das amostras de solo $(50 \mathrm{~g})$ foi ajustado a $100 \%$ da capacidade de campo, permanecendo as amostras pré-incubadas, no escuro, por sete dias, à temperatura ambiente. A seguir, foram transferidas duas subamostras de $10 \mathrm{~g}$ para recipientes plásticos, sendo uma subamostra submetida ao processo de fumigação $(\mathrm{F})$ e, a outra, mantida não fumigada (NF). A fumigação foi realizada em um dessecador, contendo um becker de vidro com 25 $\mathrm{mL}$ de clorofórmio livre de álcool, mantendo-se as amostras neste ambiente por 48 horas. Durante o mesmo período, as amostras não fumigadas foram mantidas à temperatura ambiente. Após a fumigação, as amostras F e NF foram transferidas para recipientes de vidro com fechamento hermético, permanecendo incubadas, no escuro, por 10 dias, à temperatura ambiente, sem renovação de $\mathrm{O}_{2}$ na câmara dos recipientes. No interior de cada recipiente, foi colocado um frasco com $25 \mathrm{~mL}$ de $\mathrm{NaOH}$ 0,5 M empregado para a captura do $\mathrm{C}-\mathrm{CO}_{2}$ emanado pelo solo. A quantidade de $\mathrm{C}-\mathrm{CO}_{2}$ emanada das amostras $\mathrm{F}$ e NF foi determinada por meio de titulação com $\mathrm{HCl}$ 0,1 M, usando fenolftaleína $1 \%$ como indicador. Antes da titulação, foram adicionados $10 \mathrm{~mL}$ de $\mathrm{BaCl}_{2} 0,25 \mathrm{M}$, para precipitação do carbonato, evitando interferência na titulação. A quantidade de CBM foi determinada pela diferença entre o $\mathrm{C}-\mathrm{CO}_{2}$ liberado das amostras F e NF, no período de 10 dias após a fumigação, utilizando um fator de correção $(\mathrm{kc})$ de 0,411 (Anderson \& Domsch, 1978).
O quociente microbiano ( $q \mathrm{Mic}$ ) foi calculado de acordo com Anderson \& Domsch (1989), pela relação entre CBM e COT. A respiração basal (RB) do solo consistiu do valor obtido da quantidade de $\mathrm{C}-\mathrm{CO}_{2}$ emanada das amostras não fumigadas, durante 10 dias, refletindo a atividade microbiana (Jenkinson \& Powlson, 1976). O quociente metabólico $\left(q \mathrm{CO}_{2}\right)$ foi calculado de acordo com Anderson \& Domsch (1985), pela relação entre RB e CBM.

Os resultados obtidos foram submetidos a análise de variância, sendo as médias comparadas pelo teste de Scott-Knott, a 5\% de probabilidade, utilizando o software SAEG (Fundação Arthur Bernardes, 2000).

\section{RESULTADOS E DISCUSSÃO}

O carbono orgânico total (COT) foi afetado pelos sistemas de uso do solo estudados $\quad(\mathrm{P}<0,01)$, com teores variando de 22,14 a 29,81 $\mathrm{g} \mathrm{kg}^{-1}$ de solo. Os resultados obtidos mostram que as áreas sob cana-de-açúcar e pastagem apresentaram teores similares a mata nativa, enquanto que os demais sistemas de manejo apresentaram uma tendência de redução no teor de COT, sendo esse fato mais pronunciado nas áreas sob sucessão de cultivos e consórcio de culturas (Figura 1).

Outros estudos também têm apontado eficiência das gramíneas na manutenção do COT do solo, podendo, até mesmo, em determinadas situações, ser observada uma tendência de acúmulo de $\mathrm{C}$ em relação à condição natural de cobertura, em decorrência, principalmente, da elevada geração de resíduos e intensa ciclagem de raízes nesses sistemas (Moraes et al., 2002; Andréa et al., 2004). No caso da cana-deaçúcar, além do exposto, a adoção da prática de colheita sem o uso da queima também contribuiu para esses resultados.

Com relação a área sob fruticultura, presume-se que o decréscimo no teor de COT do solo, em relação à mata, 
esteja relacionado principalmente com a baixa adição de resíduos orgânicos pela cultura do cajueiro. Já os elevados decréscimos nos valores de COT nas áreas de sucessão de cultivos e consórcio de culturas podem ser atribuídos ao sistema intensivo de exploração dessas áreas (cultivo convencional), que contribuiu para um rápido esgotamento do COT do solo, conforme argumentado por Matsuoka et al. (2003).

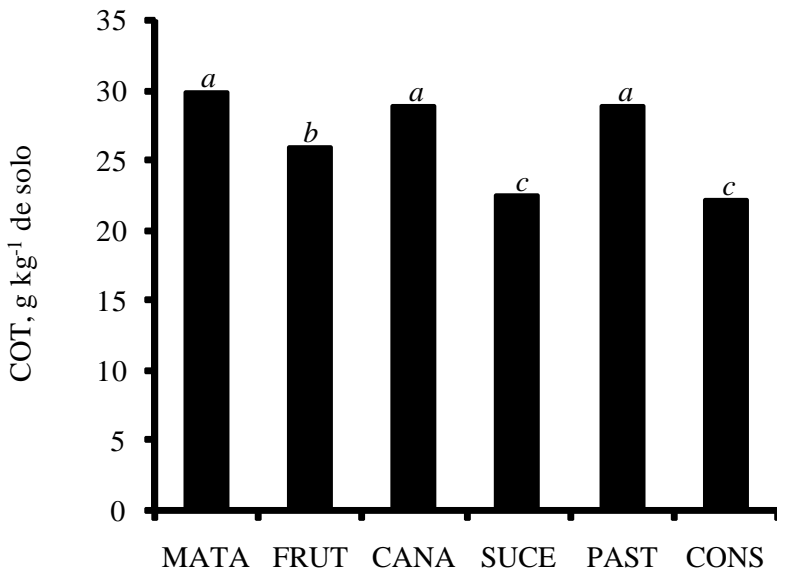

Figura 1 - Carbono orgânico total (COT) de um Latossolo Amarelo distrófico submetido a diferentes sistemas de uso em Areia-PB. MATA: mata nativa; FRUT: fruticultura; CANA: cana-de-açúcar; SUCE: sucessão de cultivos; PAST: pastagem; CONS: consórcio de culturas. Médias seguidas de letras iguais não diferem estatisticamente entre si, pelo teste de Scott-Knott a 5\% de probabilidade.

O carbono da biomassa microbiana (CBM) diferiu estatisticamente em função dos diferentes sistemas de uso do solo $(\mathrm{P}<0,01)$, apresentando valores compreendidos entre 243,54 e 359,60 $\mu \mathrm{g} \mathrm{g}^{-1}$ de solo. A mata nativa apresentou valor de CBM superior aos demais sistemas estudados, seguida pelas áreas sob cana-de-açúcar e pastagem, pela área sob fruticultura e, por fim, pelas áreas sob sucessão de cultivos e consórcio de culturas (Figura 2).

Os resultados evidenciam que, de maneira geral, o CBM tendeu a aumentar com a elevação dos valores do COT do solo, o que pode ser confirmado pela alta correlação entre esses atributos (Tabela 3), demonstrando que a quantidade de substrato orgânico disponível no sistema foi fator preponderante para as diferenças verificadas no CBM. Entretanto, considerando as áreas sob mata nativa e cultivadas com gramíneas, onde os teores de COT foram semelhantes (Figura 1), a superioridade nos valores de CBM observada na mata nativa se deve a maior diversidade de espécies vegetais presentes nesse sistema, resultando em disponibilidade de substratos orgânicos com composição variada e maior diversidade de compostos orgânicos depositados na rizosfera, o que constitui fator favorável à sobrevivência e crescimento dos diferentes grupos de microrganismos do solo (Andréa et al., 2002).

Diante do exposto, os resultados apontam que o CBM mostrou-se mais adequado que o COT para predizer a resposta do sistema edáfico às intervenções antrópicas, reforçando o seu potencial como indicador sensível para detectar modificações no solo (Andréa et al., 2002; Jordan et al., 2004; Díaz-Raviña et al., 2005), antes mesmo que os teores de matéria orgânica sejam alterados significativamente (Matsuoka et al., 2003).

Para o quociente microbiano ( $q \mathrm{Mic})$, não foi verificada diferença significativa entre os sistemas de uso, apresentando valores compreendidos entre 1,10 e $1,21 \%$ (Figura 2), indicando que o $q \mathrm{Mic}$ não discriminou as possíveis alterações decorrentes do uso agrícola do solo.

$\mathrm{O} q \mathrm{Mic}$ vem sendo mencionado como um importante indicador da qualidade da matéria orgânica do solo, sendo que variações nesse atributo podem refletir variações de matéria orgânica no sistema, a eficiência de conversão do C orgânico em $\mathrm{C}$ microbiano, perdas de $\mathrm{C}$ do solo e estabilização do $\mathrm{C}$ orgânico pelas frações minerais do solo (Tótola \& Chaer, 2002). Entretanto, alguns autores têm demonstrado que esse atributo, da forma como vem sendo determinado (relação entre CBM total e COT), pode apresentar limitações para predizer a qualidade do solo em determinadas situações. Esse fato pode ser verificado no trabalho desenvolvido por Islam \& Weil (2000a), no qual não se verificou diferença significativa para essa relação entre áreas de floresta natural, área de reflorestamento artificial (Acacia auriculiformis L. e A. minijiri L., por 5 e 7 anos, respectivamente), área de pastagem (capim napier ou Saccharum spontaneoum por 21 anos) e área explorada com diferentes culturas agrícolas (arroz, mostarda, algodão etc., durante aproximadamente 20 anos). Entretanto, os referidos autores verificaram que o emprego do CBM ativa (obtido pela técnica da respiração induzida) ao invés do CBM total na determinação do $q$ Mic se mostrou eficiente para predizer as alterações provenientes das práticas de manejo, sendo, portanto, mais indicado.

As taxas de respiração basal (RB) variaram em função dos sistemas de uso do solo estudados $(\mathrm{P}<0,01)$, com valores compreendidos entre 1,89 e $3,09 \mu \mathrm{g} \mathrm{g}^{-1} \mathrm{~h}^{-1} \mathrm{de}$ $\mathrm{C}-\mathrm{CO}_{2}$ no solo. As áreas cultivadas com cana-de-açúcar e pastagem apresentaram atividade biológica superior aos demais sistemas de uso, seguidas pela mata nativa e, por fim, pelas áreas sob sucessão de cultivos, consórcio de cultura e fruticultura (Figura 3 ). 

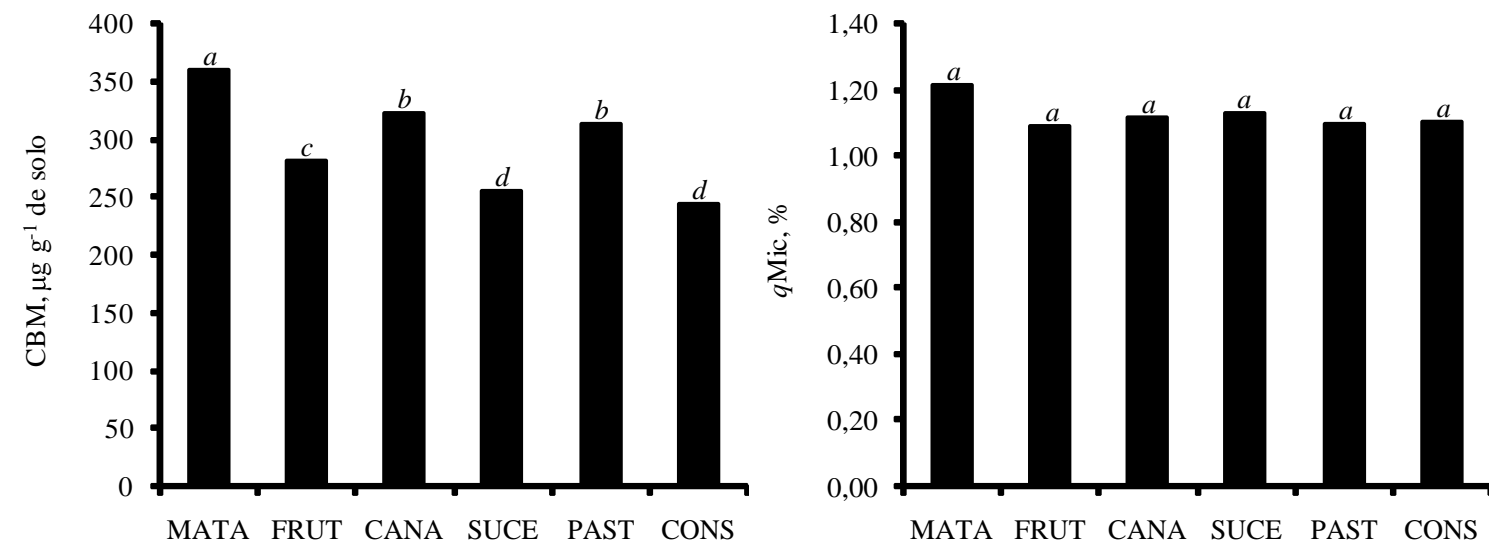

Figura 2 - Carbono da biomassa microbiana $(\mathrm{CBM})$ e quociente microbiano $(q \mathrm{Mic})$ de um Latossolo Amarelo distrófico submetido a diferentes sistemas de uso em Areia-PB. MATA: mata nativa; FRUT: fruticultura; CANA: cana-de-açúcar; SUCE: sucessão de cultivos; PAST: pastagem; CONS: consórcio de culturas. Médias seguidas de letras iguais não diferem estatisticamente entre si, pelo teste de Scott-Knott a 5\% de probabilidade.

Tabela 3 - Coeficientes de correlação de Pearson entre os indicadores biológicos estudados.

\begin{tabular}{cccccc}
\hline Atributo & COT & $\mathrm{CBM}$ & $q \mathrm{Mic}$ & $\mathrm{RB}$ & $q \mathrm{CO}_{2}$ \\
\hline $\mathrm{COT}$ & 1,000 & $0,829^{* *}$ & $-0,095^{\mathrm{ns}}$ & $0,636^{* *}$ & $0,051^{\mathrm{ns}}$ \\
$\mathrm{CBM}$ & & 1,000 & $0,476^{*}$ & $0,528^{*}$ & $-0,209^{\mathrm{ns}}$ \\
$q \mathrm{Mic}$ & & 1,000 & $-0,029^{\text {ns }}$ & $-0,418^{\text {ns }}$ \\
$\mathrm{RB}$ & & & 1,000 & $0,716^{* *}$ \\
$q \mathrm{CO}_{2}$ & & & & & 1,000 \\
\hline
\end{tabular}

COT: carbono orgânico total; CBM: Carbono da biomassa microbiana; $q \mathrm{Mic}$ : quociente microbiano; RB: respiração basal; $q \mathrm{CO}_{2}$ : quociente metabólico.

${ }_{\text {ns }},{ }^{*} \mathrm{e}^{* *}$ : não significativo e significativo a 5 e $1 \%$ de probabilidade, respectivamente, pelo teste t.
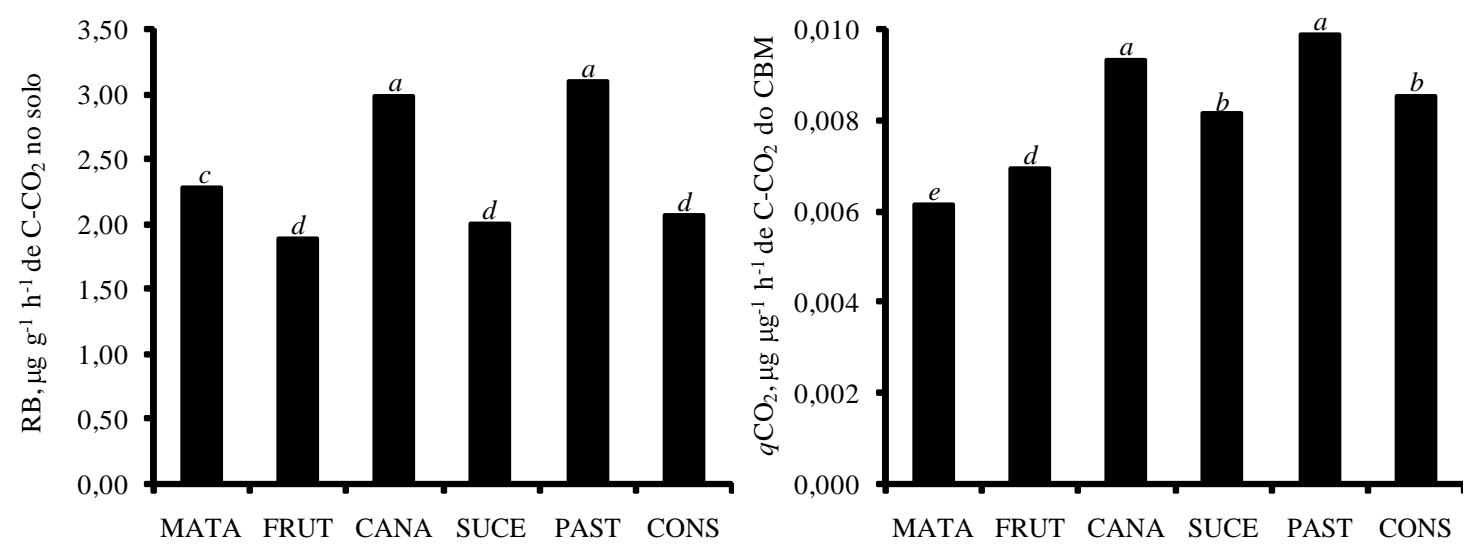

Figura 3 - Respiração basal (RB) e quociente metabólico $\left(q \mathrm{CO}_{2}\right)$ de um Latossolo Amarelo distrófico submetido a diferentes sistemas de uso em Areia-PB. MATA: mata nativa; FRUT: fruticultura; CANA: cana-de-açúcar; SUCE: sucessão de cultivos; PAST: pastagem; CONS: consórcio de culturas. Médias seguidas de letras iguais não diferem estatisticamente entre si, pelo teste de Scott-Knott a 5\% de probabilidade. 
$\mathrm{O}$ C- $\mathrm{CO}_{2}$ emanado do solo por meio da $\mathrm{RB}$ indica a intensidade com que os processos bioquímicos acontecem no ecossistema. Entretanto, a interpretação desses resultados deve ser feita com cuidado, uma vez que elevadas taxas de liberação de $\mathrm{C}-\mathrm{CO}_{2}$ no solo nem sempre indicam condições favoráveis. Isso significa, em curto prazo, maior disponibilidade de nutrientes para as plantas e, em longo prazo, perda de C orgânico do solo para atmosfera. Dessa forma, elevados valores de RB podem indicar tanto situações de distúrbio quanto de alto nível de produtividade do sistema (Islam \& Weil, 2000a). Nesse aspecto, a avaliação da atividade da biomassa microbiana pelo quociente metabólico tem se mostrado mais adequado a esses estudos (Anderson, 2003).

$\mathrm{O}$ quociente metabólico $\left(q \mathrm{CO}_{2}\right)$ foi afetado pelos sistemas de uso do solo $(\mathrm{P}<0,01)$, apresentando valores, variando de 0,0061 a $0,0099 \mu \mathrm{g}^{-1} \mathrm{~h}^{-1}$ de $\mathrm{C}-\mathrm{CO}_{2}$ do $\mathrm{CBM}$, sendo verificado comportamento similar a RB (Figura 3), o que foi constatado pela alta correlação entre essas variáveis (Quadro 3). Os resultados demonstram que as áreas sob cana-de-açúcar e pastagem apresentaram valores de $q \mathrm{CO}_{2}$ superiores aos observados para os demais sistemas de uso, seguidas pelas áreas sob sucessão de cultivos e consórcio de culturas, pela área sob fruticultura e, por fim, pela área sob mata nativa (Figura 3).

Ao propor o emprego do $q \mathrm{CO}_{2}$ como variável ecofisiográfica da atividade específica da biomassa microbiana do solo, Anderson \& Domsch (1985) predisseram que à medida que determinada população microbiana se torna mais eficiente na utilização dos recursos do ecossistema, menos $\mathrm{C}$ é perdido como $\mathrm{CO}_{2}$ pela respiração e maior proporção de $\mathrm{C}$ é incorporada às células dos microrganismos. Menores valores de $q \mathrm{CO}_{2}$ indicam agroecossistemas mais estáveis, sendo que a substituição da vegetação nativa acelera a decomposição dos resíduos com aumento do valor de $q \mathrm{CO}_{2}$ (Moreira \& Malavolta, 2004).

Nesse sentido, os elevados valores do $q \mathrm{CO}_{2}$ observados nas áreas cultivadas com gramíneas, bem como nas áreas sob sucessão de cultivos e consórcio de culturas, refletem maiores perdas de $\mathrm{C}$ nesses sistemas por unidade de CBM pelo processo respiratório, o que evidencia uma condição de estresse ou distúrbio (Islam \& Weil, 2000a,b), indicando, assim, a ocorrência de um processo degradativo desses ambientes. Esse fato provavelmente é decorrente do predomínio de microrganismos colonizadores de crescimento rápido (Baretta et al., 2005).

Os menores valores de $q \mathrm{CO}_{2}$ observados na área sob fruticultura, em relação aos demais sistemas agrícolas estudados, refletem uma condição de economia na utilização de energia, indicando que este sistema pode estar se ajustando a um novo estado de equilíbrio (Tótola \& Chaer, 2002). Os resultados deste trabalho permitem afirmar que o $q \mathrm{CO}_{2}$ indicou de forma significativa as alterações decorrentes dos diferentes usos do solo, destacando-se como um indicador bastante sensível frente às intervenções antrópicas, conforme documentado na literatura (Islam \& Weil, 2000a,b; Baretta et al., 2005).

\section{CONCLUSÕES}

Todos os sistemas agrícolas estudados promoveram perda de qualidade do solo, sendo esse fato mais pronunciado nas áreas sob manejo mais intensivo;

$\mathrm{O} \mathrm{CBM}$ e $q \mathrm{CO}_{2}$ mostraram-se bastante sensíveis às alterações decorrentes do uso agrícola do solo, apresentando grande potencial para estudos de qualidade do solo.

\section{REFERÊNCIAS BIBLIOGRÁFICAS}

ANDERSON, J.P.E.; DOMSCH, K.H. Mineralisation of bacteria and fungi in chloroform-fumigated soils. Soil Biology Biochemistry, Oxford, v.10, n.3, p.207-213, 1978

ANDERSON, J.P.E.; DOMSCH, K.H. Determination of ecophysiological maintenance carbon requirements of soil microorganisms in dormant state. Biology Fertility Soils, Berlin, v.1, n.1, p.81-89, 1985.

ANDERSON, J.P.E.; DOMSCH, K.H. Ratios of microbial biomass carbon to total organic carbon in arable soils. Soil Biology Biochemistry, Oxford, v.21, n.4, p.471-479, 1989.

ANDERSON, T.H. Microbial eco-physiological indicators to asses soil quality. Agriculture Ecosystems Environmental, Amsterdam, v.98, n.1/3, p.285-293, 2003.

ANDRÉA, A.F. d'; SILVA, M.L.N.; CURI, N.; GUILHERME, L.R.G. Estoques de carbono e nitrogênio e formas de nitrogênio mineral em um solo submetido a diferentes sistemas de manejo. Pesquisa Agropecuária Brasileira, Brasília, v.39, n.2, p.179-186, 2004.

ANDRÉA, A.F. d'; SILVA, M.L.N.; CURI, N.; SIQUEIRA, J.O.; CARNEIRO, M.A.C. Atributos biológicos indicadores da qualidade do solo em sistemas de manejo na região do cerrado no sul do Estado de Goiás. Revista Brasileira de Ciência do Solo, Viçosa, v.26, n.4, p.913923, 2002. 
BARETTA, D.; SANTOS, J.C.P.; FIGUEIREDO, S.R.; KLAUBERG-FILHO, O. Efeito do monocultivo de Pinus e da queima do campo nativo em atributos biológicos do solo no Planalto Sul Catarinense. Revista Brasileira de Ciência do Solo, Viçosa, v.29, n.5, p.715-724, 2005.

BRASIL. Ministério da Agricultura. Equipe de pedologia e fertilidade do solo. Divisão de Agrologia - SUDENE.

Levantamento exploratório: reconhecimento de solos do estado da Paraíba. Rio de Janeiro: MA/CONTA/USAID/ SUDENE, 1972. 670p. (Boletim técnico, 15).

DÍAZ-RAVIÑA, M.; BUENO, J.; GONZÁLEZ-PRIETO, S.J.; CARBALLAS, T. Cultivation effects on biochemical properties, $\mathrm{C}$ storage and ${ }^{15} \mathrm{~N}$ natural abundance in the 0 $5 \mathrm{~cm}$ layer of an acidic soil from temperate humid zone. Soil Tillage Research, Amsterdam, v.84, n.2, p.216-221, 2005.

EMPRESA BRASILEIRA DE PESQUISA

AGROPECUÁRIA. Centro Nacional de Pesquisa de Solos. Manual de métodos de análise de solos. 2.ed. Rio de Janeiro, 1997. 212p. (Embrapa-CNPS. Documentos, 1).

FUNDAÇÃO ARTHUR BERNARDES. SAEG - Sistema para Análises Estatísticas. Versão 8.0. Viçosa, MG, 2000.

HARRIS, J.A. Measurements of the soil microbial community for estimating the success of restoration. European Journal Soil Science, Oxford, v.54, n.4, p.801808, 2003.

ISLAM, K.R.; WEIL, R.R. Land use effects on soil quality in a tropical forest ecosystem of Bangladesh. Agriculture Ecosystems Environmental, Amsterdam, v.79, n.1, p.9-16, 2000a.

ISLAM, K.R.; WEIL, R.R. Soil quality indicator properties in mid-Atlantic soils as influenced by conservation management. Journal Soil Water Conservation, Ankeny, v.55, n.1, p.69-78, 2000b.

JENKINSON, D.S.; POWLSON, D.S. The effects of biocidal treatments on metabolism in soil: 5., amethod for measuring soil biomass. Soil Biology Biochemistry, Oxford, v.8, n.3, p.209-213, 1976.

JOHNSON, D.; BOOTH, R.E.; WHITELEY, A.S.; BAILEY, M.J.; READ, D.J.; GRIME, J.P.; LEAKE, J.R. Plant community composition affects the biomass, activity and diversity of microorganisms in limestone grassland soil. European Journal Soil Science, Oxford, v.54, n.4, p.671-677, 2003.

JORDAN, D.; MILES, R.J.; HUBBARD, V.C.; LORENZ, T. Effect of management practices and cropping systems on earthworm abundance and microbial activity in Sanborn Field: a 115-year-old agricultural field. Pedobiologia, Jena, v.48, n.2, p.99-110, 2004.

MATSUOKA, M.; MENDES, I.C.; LOUREIRO, M.F.R. Biomassa microbiana e atividade enzimática em solos sob vegetação nativa e sistemas agrícolas anuais e perenes na região de Primavera do Leste (MT). Revista Brasileira de Ciência do Solo, Viçosa, v.27, n.3, p.425-433, 2003.

MORAES, J.F.L. de; NEILL, C.; VOLKOFF, B.; CERRI, C.C.; MELILLO, J.; LIMA, V.C.; STEUDLER, P.A. Soil carbon and nitrogen stocks following forest conversion to pasture in the Western Brazilian Amazon Basin. Acta Scientiarum, Maringá, v.24, n.5, p.1369-1376, 2002.

MOREIRA, A.; MALAVOLTA, E. Dinâmica da matéria orgânica e da biomassa microbiana em solo submetido a diferentes sistemas de manejo na Amazônia Ocidental. Pesquisa Agropecuária Brasileira, Brasília, v.39, n.11, p.1103-1110, 2004.

NANNIPIERI, P.; ASCHER, J.; CECCHERINI, M.T.; LANDI, L.; PIETRAMELLARA, G.; RENELLA, G. Microbial diversity and soil functions. European Journal Soil Science, Oxford, v.54, n.4, p.655-670, 2003.

POKARZHEVSKII, A.D.; STRAALEN, N.M. van; ZABOEV, D.P.; ZAITSEV, A.S. Microbial links and element flows in nested detrital food-webs.

Pedobiologia, Jena, v.47, n.3, p.213-224, 2003.

SANGHA, K.K.; MIDMORE, D.J.; ROLFE, J.; JALOTA, R.K. Tradeoffs between pasture production and plant diversity and soil health attributes of pasture systems of Central Queensland, Australia. Agriculture Ecosystems Environmental, Amsterdam, v.111, n.1, p.93-103, 2005.

TÓTOLA, M.R.; CHAER, G.M. Microorganismos e processos microbiológicos como indicadores de qualidade dos solos. In: ALVAREZ, V.H.; SCHAEFER, C.E.G.R.; BARROS, N.F.; MELLO, J.W.V.; COSTA, L.M. (Eds.). Tópicos em ciência do solo. Viçosa, MG: SBCS, 2002. v.2, p.195-276. 